\title{
Diagnostic accuracy and reproducibility of optical flow ratio for functional evaluation of coronary stenosis in a prospective series
}

Juan Luis Gutiérrez-Chico ${ }^{1 *}$, Yundai $\mathrm{Chen}^{2 *}$, Wei $\mathrm{Yu}^{3 *}$, Daixin $\mathrm{Ding}^{3}$, Jiayue Huang ${ }^{3}$, Peng Huang ${ }^{3}$, Jing Jing ${ }^{2}$, Miao $\mathrm{Chu}^{1,3}$, Peng $\mathrm{Wu}^{3}$, Feng $\mathrm{Tian}^{2}$, Bo $\mathrm{Xu}^{4}$, Shengxian $\mathrm{Tu}^{3}$

${ }^{1}$ Department of Interventional Cardiology, Campo de Gibraltar Health Trust, Algeciras, Spain

${ }^{2}$ Department of Cardiology, PLA General Hospital, Beijing, China

${ }^{3}$ Biomedical Instrument Institute, School of Biomedical Engineering,

Shanghai Jiao Tong University, Shanghai, China

${ }^{4} \mathrm{Fu}$ Wai Hospital, National Center for Cardiovascular Diseases,

Chinese Academy of Medical Sciences, Beijing, China

\begin{abstract}
Background: Evaluating prospectively the feasibility, accuracy and reproducibility of optical flow ratio (OFR), a novel method of computational physiology based on optical coherence tomography (OCT).

Methods and results: Sixty consecutive patients (76 vessels) underwent prospectively OCT, angiography-based quantitative flow ratio (QFR) and fractional flow ratio (FFR). OFR was computed offline in a central core-lab by analysts blinded to FFR. OFR was feasible in $98.7 \%$ of the lesions and showed excellent agreement with FFR (ICC $a=0.83, r=0.83$, slope $=0.80$, intercept $=0.17$, kappa $=0.84$ ). The area under curve to predict an FFR $\leq 0.80$ was 0.95 , higher than for $Q F R(0.91, p=0.115)$ and for minimal lumen area $(0.64, p<0.001)$. Diagnostic accuracy, sensitivity, specificity, positive predictive value, negative predictive value, positive likelihood ratio and negative likelihood ratio were $93 \%, 92 \%$, 93\%, 88\%, 96\%, 13.8, 0.1, respectively. Median time to obtain OFR was 1.07 (IQR: 0.98-1.16) min, with excellent intraobserver and interobserver reproducibility (0.97 and 0.95, respectively). Pullback speed had negligible impact on OFR, provided the same coronary segment were imaged (ICCa $=0.90$, kappa $=0.697$ ).

Conclusions: The prospective computation of OFR is feasible and reproducible in a real-world series, resulting in excellent agreement with FFR, superior to other image-based methods. (Cardiol J 2020; 27, 4: 350-361)
\end{abstract}

Key words: optical flow ratio, optical coherence tomography, fractional flow reserve, coronary heart disease

\section{Editorial p. 345}

\section{Introduction}

The utility and safety of physiology-guided percutaneous coronary intervention (PCI) has been proved in several large randomized trials and in different clinical scenarios, consistently translating into better clinical outcomes than merely angiography-guided PCI [1-3]. Notwithstanding this compelling evidence, the penetrance of physiology in clinical routine is still low [4]. Data from a National German registry reported that fractional flow reserve (FFR) was used in only $3.2 \%$ of patients undergoing coronary angiography [5]. The

Address for correspondence: Shengxian Tu, PhD, FACC, FESC, Room 123, Med-X Research Institute, Shanghai Jiao Tong University, No. 1954, Hua Shan Road, Shanghai 200030, China, tel: +86 21 62932631, fax: +86 2162932156 ,

e-mail: sxtu@sjtu.edu.cn

Received: 15.05.2020 Accepted: 16.05.2020

*The first three authors equally contributed. 
reasons for this evidence-reality mismatch rely on the need for an additional wire, often with limited steerability and navigability, the eventual infusion of adenosine prolonging the procedure and producing discomfort to the patient, or on restrictions in the reimbursement [6]. Most health systems indeed reimburse only one adjuvant tool for PCI, either FFR or an invasive imaging tool in the best of cases, but not both. Thus, some operators give priority to imaging if they anticipate a likely PCI, because imaging can be more useful to assess the PCI result or to guide optimisation.

Among all invasive imaging modalities, optical coherence tomography (OCT) has the highest resolution and enables an accurate assessment of expansion and apposition of the stent [7-9], thus arising as a paramount tool for PCI optimisation [10]. Nonetheless, its ability to assess the functional significance of a stenosis is negligible hitherto [11]. An OCT-based morphofunctional method enabling both accurate estimation of coronary physiology and high resolution imaging for PCI optimisation within a single catheter [12] could spare time and weariness to the interventional team, discomfort to the patient, whilst being instrumental to overcome the reimbursement restraints that are preventing physiology and imaging to expand up to the boundaries where current evidence recommends their use $[6,13]$.

A novel OCT-based morphofunctional computational method dubbed optical flow ratio (OFR) has been recently developed, combining an estimation of physiology with high resolution imaging in the same OCT pullback [14-16]. OFR showed optimal agreement with FFR in post-hoc retrospective analysis $[15,16]$. Furthermore, OFR might improve the accuracy of angiography-based morphofunctional computational methods [16-18] by overcoming the intrinsic limitations of angiography, namely overlapping vessels, foreshortening and calcium interfering with an accurate edge detection of the vessel lumen, because OCT renders the most accurate lumen reconstruction currently available.

We aimed to assess the feasibility and accuracy of OFR in a prospective cohort of consecutive unselected patients, compared with other morphofunctional approaches.

\section{Methods}

\section{Study design}

This was a prospective and multicenter study to evaluate the feasibility, diagnostic accuracy and reproducibility of OFR analysis in a real-world cohort of consecutive unselected patients, taking FFR as reference standard. Secondary objectives of the study were the comparison of the diagnostic accuracy of OFR (an OCT-based computational method) vs. quantitative flow ratio (QFR, an angiography-based computational method) and the potential impact of OCT pullback speed on the diagnostic accuracy of OFR. Finally, the diagnostic accuracy of computational methods was compared vs. the approach of estimating severity by means of a minimal lumen area (MLA) cutoff.

The study was performed at two centers: Campo de Gibraltar Health Trust, Algeciras (Spain) and Chinese PLA General Hospital, Beijing (China), complying with the principles of good clinical practice and with the Declaration of Helsinki for investigation in human beings. The study protocol was approved by the institutional review boards of these two hospitals. All patients provided written informed consent.

\section{Study population}

Patients with coronary artery disease and a clinical indication for FFR measurement (40\% to $90 \%$ diameter stenosis by visual estimation, without evidence of ischemia by non-invasive tests) were included into the study in the two participating centers between November 2018 and April 2019, with varying enrolment periods in each center depending on the corresponding initiation dates. Exclusion criteria comprised aorta-ostial lesion, prior coronary artery bypass grafting, contraindication for intravenous adenosine, hemodynamic or electrical instability, moderate or severe valvular heart disease, acute coronary syndrome $<72 \mathrm{~h}$ depending on the target vessel and the presence of a chronic total occlusion in any other coronary vessel.

\section{Invasive coronary angiography}

Coronary angiography was performed through femoral, radial or ulnar approaches, with a $\geq 6 \mathrm{~F}$ catheter. After administration of intracoronary nitroglycerine, two angiographic projections of the target vessel at least $25^{\circ}$ apart were acquired at $\geq 25$ frames/s by a flat panel system, including the whole coronary artery from ostium to distal vessel, minimising vessel overlap and foreshortening, to warrant the highest quality in QFR analysis.

\section{OCT acquisition}

An OCT Dragonfly ${ }^{\mathrm{TM}}$ catheter (Abbott, St. Paul, Minnesota, USA) was advanced at least $15 \mathrm{~mm}$ distally to the stenosis. Then a pullback 
was acquired with an OPTIS ${ }^{\mathrm{TM}}$ Fourier-domain OCT system at a rotation speed of $180 \mathrm{~Hz}$ and a pullback speed of $36 \mathrm{~mm} / \mathrm{s}$ with non-occlusive technique [19], including the whole vessel up to the guiding catheter. The minimal amount of contrast required to ensure optimal quality was calculated by means of a validated formula [20]. The cases from one center were similarly acquired with a F-1 system and a T-1 catheter (Forssmann Medical Co. Ltd., Nanjing, China), both with analogous technical characteristics to the abovementioned system except for a rotation speed of $100 \mathrm{~Hz}$ and a pullback speed of $18 \mathrm{~mm} / \mathrm{s}$.

Lesions imaged with the Dragonfly ${ }^{\mathrm{TM}}$ catheter (Abbott, St. Paul, Minnesota, USA) underwent a second pullback at $18 \mathrm{~mm} / \mathrm{s}$ following the same protocol, unless clinically contraindicated due to excessive amount of contrast or instability of the patient. In case of sequential lesions or diffuse coronary heart disease, two consecutive OCT pullbacks could be acquired to cover the whole stenotic segment.

\section{FFR measurement}

An Aeris pressure wire (Abbott, St. Paul, Minnesota, USA) was used for FFR measurement. Distal pressure was equalised with the aortic pressure at the tip of the guiding catheter, filled with saline, before advancing the wire distally to the lesion. Hyperemia was induced by means of a continuous intravenous infusion of $140 \mu \mathrm{g} / \mathrm{kg} / \mathrm{min}$ adenosine and FFR was then recorded as the minimal distal coronary pressure to aortic pressure ratio $(\mathrm{Pd} / \mathrm{Pa})$ value in maximal stable hyperemia. The pressure transducer was then pulled back again to the tip of the guiding catheter: a pressure drift of $\pm 3 \mathrm{mmHg}$ was considered acceptable. If the pressure drift exceeded this margin, the FFR recording was repeated. For sequential lesions, the FFR at the most distal position was used for comparison with OFR.

\section{OFR and QFR analysis}

Optical coherence tomography recordings were centrally analysed in a core-lab setting (CardHemo, Med-X Research Institute, Shanghai Jiao Tong University, China). OFR analysis was performed in all OCT pullbacks unless the OCT catheter were occlusive, thus precluding an optimal visualization of the vessel distally to the stenosis. However, OFR was excluded for comparison vs. FFR if any of the following was present: 1) Vessel spasm or injury during OCT acquisition; 2) Myocardial bridge in the angiography; 3) OCT pullback not covering the entire lesion; 4) Intracoronary thrombus.

Optical flow ratio analysis and QFR analysis were separately performed by two independent analysts in a blinded fashion, using the OctPlus software (version 1.0) and the AngioPlus system, respectively (both from Pulse Medical Imaging Technology, Shanghai, China), following a methodology previously described [14, 15, 21, 22]. Briefly, for OFR computation, lumen contours were automatically delineated in the OCT pullback to render a tubular three-dimensional (3D) reconstruction of the lumen. The ostia of side branches were automatically detected and outlined to calculate the corresponding cross-sectional areas [23]. Manual edition was allowed in case of misleading contour detection. The reference lumen area at the distal part of the bifurcation was then derived according to the principles of fractal geometry [24]. Finally, the reference lumen area was multiplied by a fixed flow velocity of $0.35 \mathrm{~m} / \mathrm{s}$ to obtain the hypothetic volumetric flow used as boundary condition for the OFR algorithm $[15,16]$, thus enabling the computation of OFR (i.e., the estimated FFR value) along the reconstructed vessel $[14,15]$. For sequential stenosis requiring two different pullbacks, both pullbacks were combined using a fiduciary landmark. Considering OFR 1 as the OFR calculated in the proximal pullback at the fiduciary landmark, and considering OFR2 as the OFR in the distal pullback after excluding the vessel proximal to the fiduciary landmark, the combined OFR was calculated as OFR1 + OFR2 - 1.0.

For QFR analysis, the lumen contour of the coronary vessel was automatically detected in two angiographic projections for 3D angiographic reconstruction. Manual correction was allowed in case of suboptimal image quality, following a standard operational procedure $[18,21]$. The contrast flow model was used for the computations, calculating the coronary flow as the product of reference lumen area and contrast velocity estimated by Thrombolysis in Myocardial Infarction (TIMI) frame count [18]. The resulting coronary flow was used as boundary condition for the QFR algorithm to compute the estimated FFR value along the reconstructed vessel $[18,21,22]$.

Figure 1 shows an example of OFR and QFR analysis. OFR and QFR were compared head-to-head vs. FFR, taking the respective values obtained at the same position where FFR was recorded.

Optical flow ratio analysis was repeated by the first OFR analyst 1 month later and by a second 


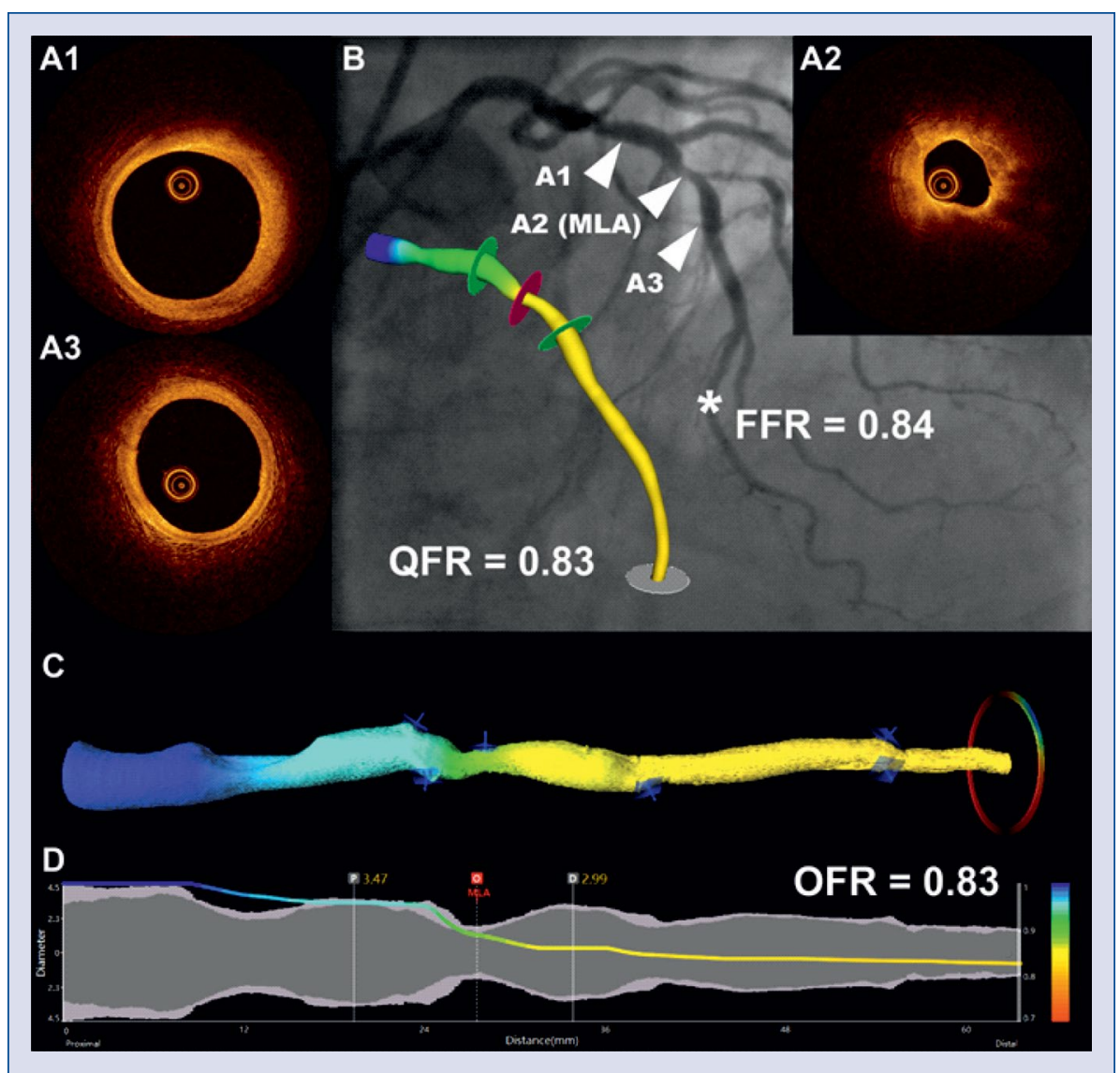

Figure 1. Computation of optical flow ratio (OFR) and quantitative flow ratio (QFR) in an intermediate stenosis in the left anterior descending artery. Cross-sections A1-A3 correspond to the angiography positions showed in panel B (arrow heads), with $\mathbf{A} 2$ as minimal lumen area (MLA). Fractional flow reserve (FFR) measured at a distal position (B, ${ }^{*}$ ) was 0.84 . The computed QFR (B) and optical flow ratio (OFR) (C) along the vessel are color-coded and superimposed on a three-dimensional reconstruction, both with a value of 0.83 at the marked point $\left(^{*}\right)$. The software renders a virtual pressure pullback within the coronary artery (D) for optimal co-registration between pressure-drop and anatomy.

analyst, following the same operational procedure and blinded to each other's results and to previously calculated OFR. The analysis time for OFR was recorded, starting from the moment in which OCT images were uploaded into the computation software and ending when the OFR value was finally obtained.

\section{Statistical analysis}

Descriptive statistics of continuous variables were reported as mean \pm standard deviation (SD) or as median (quartiles) as appropriate, while those of categorical variables were presented as counts (percentages). Continuous variables were compared with unpaired t-test or Mann-Whitney $\mathrm{U}$ test, as appropriate, whilst categorical variables were compared with Fisher's exact test. Correlation was evaluated using Pearson's correlation coefficient and linear regression analysis, taking
FFR as standard reference. Constant bias was evaluated as the deviation of the intercept from 0 , whilst proportional bias was evaluated as the deviation of the slope from 1 in the linear regression. Agreement of the different continuous parameters in the study was reported as intraclass correlation coefficient for the absolute value (ICCa) and by means of Bland-Altman plots. The agreement between OFR, QFR and FFR as dichotomous variables (significant $\leq 0.80$, non-significant $>0.80$ ) was reported as kappa coefficient. Diagnostic accuracy, sensitivity (Se), specificity (Sp), positive and negative predictive values $(+\mathrm{PV},-\mathrm{PV})$, positive and negative likelihood ratios ( $+\mathrm{LR},-\mathrm{LR}$ ) were reported. The diagnostic performance was assessed by the area under the curve (AUC) in the receiver-operating characteristic (ROC) curve. An optimal cutoff value of the MLA in OCT to detect a hemodynamically significant FFR was calculated 


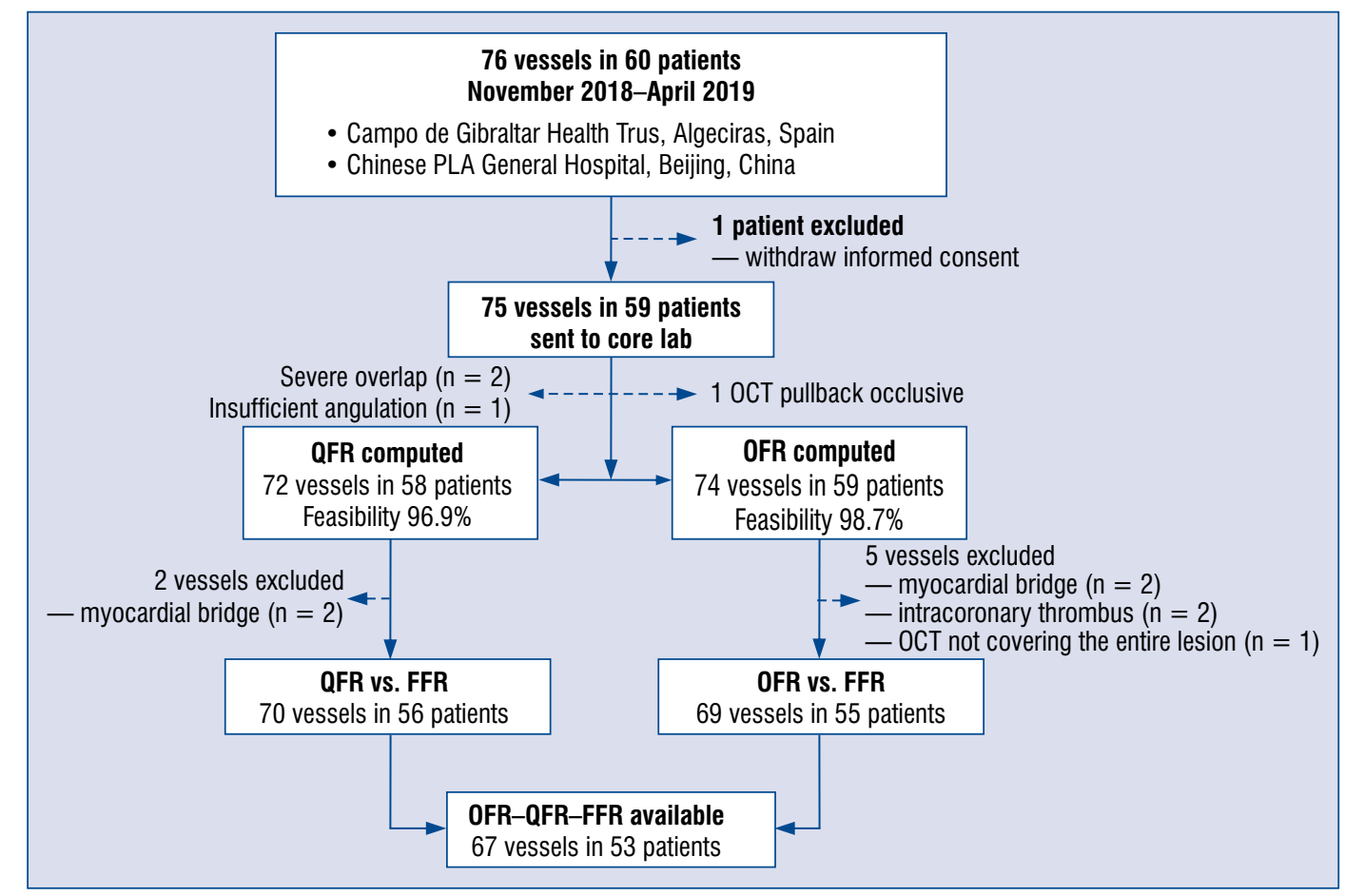

Figure 2. Study flow chart; FFR — fractional flow reserve; OCT — optical coherence tomography; OFR — optical flow ratio; QFR - quantitative flow ratio.

with the Youden index in the series and the corresponding AUC calculated. AUC of the different diagnostic methods were compared with the Delong method using MedCalc 14.12 (MedCalc Software, Ostend, Belgium). For all other statistics the IBM SPSS 24.0 software package (SPSS Inc., Chicago, Illinois) was employed.

Sample size was calculated by Liao's method for studies of agreement [25], assuming an AUC of 0.93 , as previously reported [15], and an $\alpha$ error 0.05 . Under these premises, $\mathrm{n} \geq(\log [1-0.93] / \log$ [1-0.05]), resulting in a minimal sample size of 52 patients for a reliable estimation of the AUC. Accounting for a $10 \%$ of cases unsuitable for analysis, the sample size increased to 58 patients.

\section{Results}

\section{Baseline clinical and lesion characteristics}

Sixty patients ( 76 vessels) were prospectively enrolled into the study, but 1 patient withdrew informed consent before FFR acquisition. OCT was occlusive in 1 vessel, but OFR could be computed in the remaining 74 vessels (feasibility $98.7 \%$ ). Five vessels were however excluded by the corelab for comparison vs. FFR due to myocardial bridge $(\mathrm{n}=2)$, intracoronary thrombus $(\mathrm{n}=2)$ or incomplete OCT imaging of the lesion $(\mathrm{n}=1)$ (Fig. 2), resulting in 55 patients (69 vessels) for paired comparison vs. FFR. OCT was acquired at different pullback speeds in 27 vessels, as previously described. QFR computation was successful in 72 out of 75 vessels (feasibility $96.0 \%$ ). Unsuccessful QFR computations were due to severe overlap at the interrogated vessel $(n=2)$ or insufficient difference in angulation between the angiographic projections $(\mathrm{n}=1)$. Two vessels were discarded in the corelab due to myocardial bridge, resulting in 56 patients and 70 vessels for paired comparison QFR vs. FFR.

Baseline characteristics are presented in Tables 1 and 2. Most of the cases corresponded to stable coronary heart disease $(72.9 \%$, comprising stable angina, silent ischemia or a prognostic indication), although there were also acute coronary syndromes older than $72 \mathrm{~h}$ and provoked by morphologically stable plaques (Table 1 ). The left anterior descending (LAD) artery was the most commonly interrogated vessel $(52.0 \%)$, followed by the right coronary artery (RCA) $(30.7 \%)$. A significant proportion of lesions corresponded to bifurcations $(54.7 \%)$ or sequential stenosis $(46.7 \%)$.

The average FFR value was $0.83 \pm 0.09$. In $26(34.7 \%)$ lesions FFR was $\leq 0.80$ and $33(44.0 \%)$ 
Table 1. Baseline clinical characteristics (patients, $\mathrm{n}=59$ ).

\begin{tabular}{lc}
\hline Age [years] & $63.4 \pm 10.1$ \\
Women & $9(15.3 \%)$ \\
Body mass index $\left[\mathrm{kg} / \mathrm{m}^{2}\right]$ & $28.1[25.4,30.6]$ \\
Diabetes mellitus & $23(39.0 \%)$ \\
Hypertension & $40(67.8 \%)$ \\
Hyperlipidemia & $31(52.5 \%)$ \\
Current smoker & $17(28.8 \%)$ \\
Family history of CAD & $6(10.2 \%)$ \\
Previous PCl & $40(67.8 \%)$ \\
Previous CABG & $2(3.4 \%)$ \\
Previous MI & $28(47.5 \%)$ \\
Clinical presentation: & \\
Silent ischemia/prognostic indication & $32(54.2 \%)$ \\
Stable angina & $11(18.6 \%)$ \\
Unstable angina & $12(20.3 \%)$ \\
NSTEMI & $4(6.8 \%)$ \\
Patients with FFR measurement & $15(25.4 \%)$ \\
in $>1$ vessel & \\
\hline
\end{tabular}

Data are presented as mean \pm standard deviation, median (Q1-Q2) or number (\%), as appropriate; CAD - coronary artery disease; CABG - coronary artery bypass surgery; NSTEMI — non-ST-elevation myocardial infarction; $\mathrm{MI}$ - myocardial infarction; $\mathrm{PCl}-$ percutaneous coronary intervention; FFR - fractional flow reserve

Table 2. Baseline vessel characteristics (lesions, $\mathrm{n}=75)$.

\begin{tabular}{lc}
\hline Target vessel: & \\
Left anterior descending & $39(52.0 \%)$ \\
Diagonal & $5(6.7 \%)$ \\
Left circumflex & $4(5.3 \%)$ \\
Obtuse marginal & $2(2.7 \%)$ \\
Ramus intermedius & $2(2.7 \%)$ \\
Right coronary artery & $23(30.7 \%)$ \\
Bifurcation lesions & $41(54.7 \%)$ \\
Sequential stenosis & $35(46.7 \%)$ \\
Fractional flow reserve (FFR) data: & \\
FFR & $0.83 \pm 0.09$ \\
FFR $\leq 0.80$ & $26(34.7 \%)$ \\
$0.75 \leq$ FFR $\leq 0.85$ & $33(44.0 \%)$ \\
\hline
\end{tabular}

Data are presented as mean \pm standard deviation or number $(\%)$, as appropriate.

lesions had a central FFR value between 0.75 and 0.85 (Table 2 ).

\section{Agreement between OFR and FFR}

Optical flow ratio had the same average value as FFR $0.83 \pm 0.09$. In the linear regression analy- sis, taking FFR as reference (y), OFR showed good correlation $(\mathrm{r}=0.83)$, with neither proportional (slope $=0.80)$ nor constant bias (intercept $=0.17$ ) (Fig. 3). Bland-Altman analysis is presented in Figure 3, showing no significant difference between OFR and FFR (mean difference $=0.00$, SD of the difference 0.05). The agreement between both methods was excellent (ICCa $=0.83 ; 95 \%$ confidence interval [CI]: 0.74-0.89).

The agreement between OFR and FFR as dichotomous variables was also excellent (kappa $=0.84 ; 95 \%$ CI: 0.71-0.98).

\section{Agreement between QFR and FFR}

In linear regression, taking FFR as reference $(y)$, QFR showed good correlation $(r=0.78)$, although sensibly more deviated from unbiasedness (slope $=$ $=0.61$, intercept $=0.33$ ) (Fig. 3). Bland-Altman analysis is presented in Figure 3, showing no significant difference between QFR and FFR (mean difference $=0.00$, SD of the difference 0.07). The agreement between both methods was very good $(\mathrm{ICCa}=0.75 ; 95 \%$ CI: 0.63-0.84).

The agreement between QFR and FFR as dichotomous variables was excellent (kappa $=0.78$; 95\% CI: 0.63-0.93).

\section{Diagnostic performance of OFR, QFR and OCT-MLA}

The diagnostic accuracy of OFR was $93 \%$ (95\% CI: 86-99), with 22 true positives, 42 true negatives, 3 false positives and 2 false negatives, corresponding to a sensitivity, specificity, positive predictive value (PPV), negative predictive value (NPV), positive likelihood ratio (+LR) and negative likelihood ratio (-LR) of $92 \%$ (95\% CI: 73-99), 93\% (95\% CI: 82-99), 88\% (95\% CI: 69-98), 96\% (95\% CI: 85-99), 13.8 (95\% CI: 4.6-41.3), and 0.1 (95\% CI: $0.0-0.3$ ). This diagnostic performance was slightly better than that of QFR: diagnostic accuracy, sensitivity, specificity, PPV, NPV, +LR, and -LR of 90\% (95\% CI: 83-97), 91\% (95\% CI: 72-99), 89\% (95\% CI: $77-97), 81 \%$ (95\% CI: $61-93), 96 \%$ (95\% CI: 85-99), 8.6 (95\% CI: 3.7-19.8), and 0.1 (95\% CI: $0.0-0.4$ ), respectively (Table 3 ).

The AUC of OFR was 0.95 , tendentially higher than that of QFR (0.91, p = 0.115) and significantly higher than that of OCT-derived MLA (0.64, $\mathrm{p}<0.001$ ), taking for the latter the best cut-off value found in the ROC analysis of the series $\left(1.63 \mathrm{~mm}^{2}\right)$ (Fig. 4).

In the subgroup analysis (Fig. 5), the diagnostic accuracy of OFR was independent of the coronary vessel (LAD 92\%; 95\% CI: 83-100, 

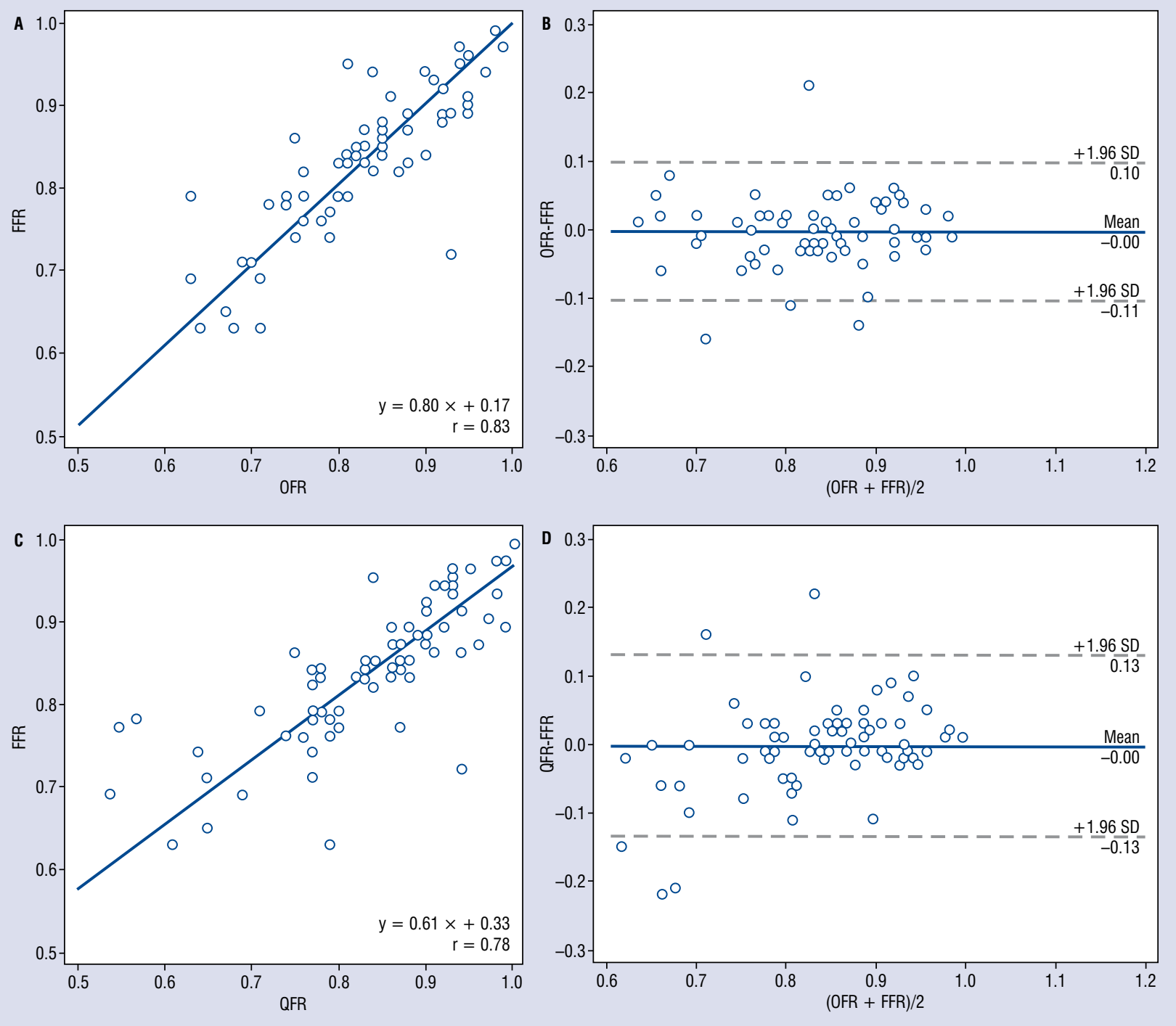

Figure 3. Correlation and agreement between fractional flow reserve (FFR) and optical flow ratio (OFR). Agreement between OFR and FFR: lineal regression (A) and Bland-Altman plot (B). Agreement between QFR and FFR: lineal regression (C) and Bland-Altman plot (D); SD — standard deviation.

Table 3. Diagnostic performance of optical flow ratio, quantitative flow ratio (QFR) and optical coherence tomography (OCT)-derived minimum lumen area (MLA) to identify fractional flow reserve $\leq 0.80$.

\begin{tabular}{|c|c|c|c|}
\hline & OFR $\leq 0.80$ & QFR $\leq \mathbf{0 . 8 0}$ & $\mathrm{MLA} \leq 1.63 \mathrm{~mm}^{2}$ \\
\hline Accuracy & 93 (86-99) & 90 (83-97) & $68(57-79)$ \\
\hline Sensitivity & 92 (73-99) & 91 (72-99) & $54(33-74)$ \\
\hline Specificity & 93 (82-99) & 89 (77-97) & 76 (61-87) \\
\hline PPV & $88(69-98)$ & 81 (61-93) & $54(33-74)$ \\
\hline NPV & $96(85-99)$ & 96 (85-99) & $76(61-87)$ \\
\hline$+\mathrm{LR}$ & $13.8(4.6-41.3)$ & $8.6(3.7-19.8)$ & $2.2(1.2-4.2)$ \\
\hline -LR & $0.1(0.0-0.3)$ & $0.1(0.0-0.4)$ & $0.6(0.4-1.0)$ \\
\hline
\end{tabular}

Data for + LR and -LR presented as ratio $(95 \% \mathrm{Cl})$ or for the rest of parameters as $\%(95 \% \mathrm{Cl})$; $\mathrm{Cl}$ - confidence interval; NPV - negative predictive value; PPV - positive predictive value; +LR - positive likelihood ratio; - LR - negative likelihood ratio 


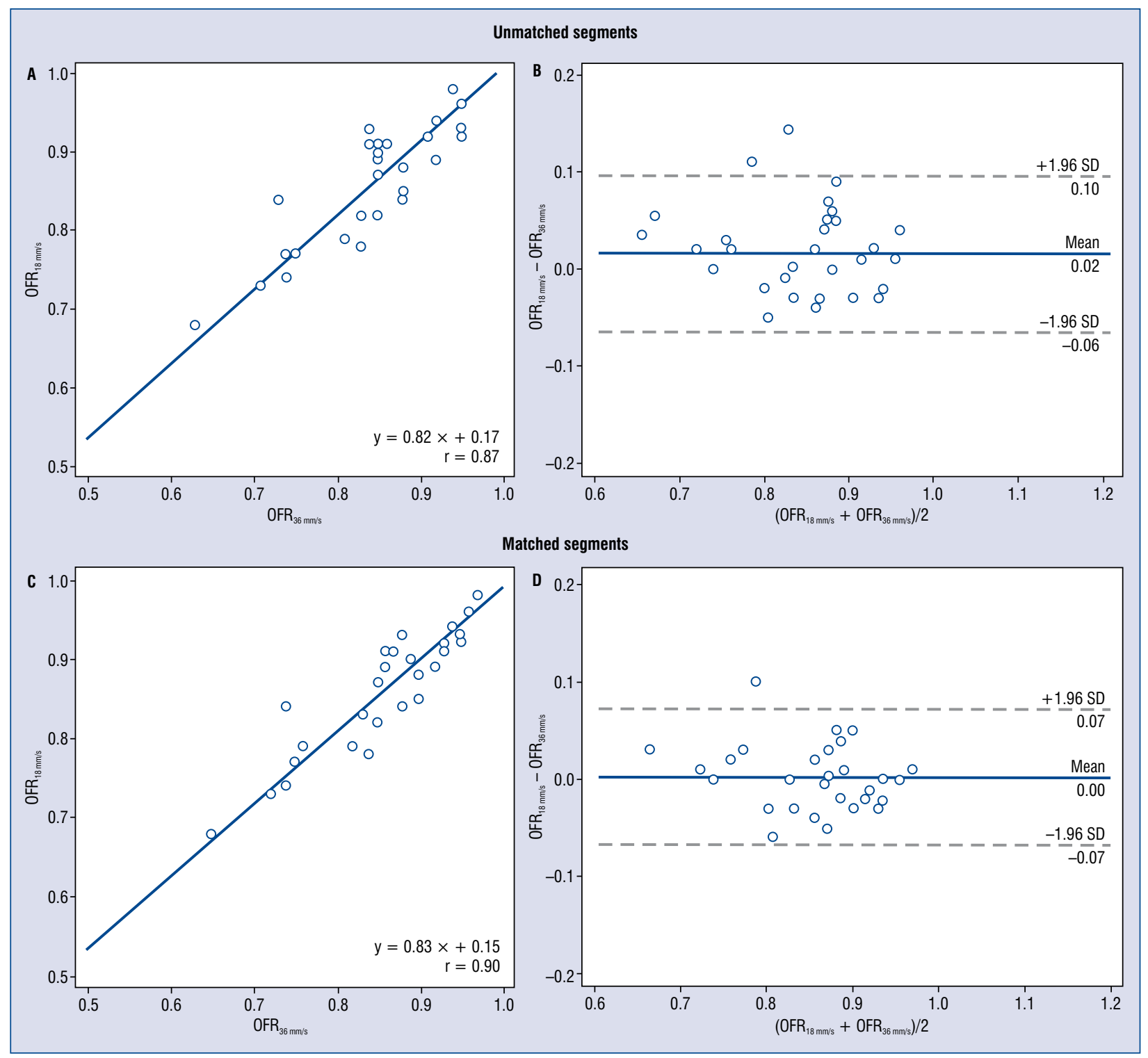

Figure 6. Correlation and agreement between optical flow ratio (OFR) acquired at different pullback speeds. A. Correlation and lineal regression of OFR acquired at $36 \mathrm{~mm} / \mathrm{s}$ (abscises) and OFR acquired at $18 \mathrm{~mm} / \mathrm{s}$ (ordinates); B. Bland-Altman plot for the agreement; Lower panels present the same lineal regression (C) and Bland-Altman plot (D) after matching the same coronary segment in the optical coherence tomography pullbacks acquired at different pullback speeds.

superior to angiography-based morphofunctional methods and significantly better than using a MLA cut-off; 4) The pullback speed has negligible effect on the computation of OFR, provided the pullbacks are obtained on the same coronary segment.

To the best of our knowledge, this is the first study to prove the feasibility of OFR prospectively in a real-world scenario, confirming the excellent diagnostic performance of previous retrospective reports $[15,16]$. Actually, the diagnostic accuracy in this prospective series was even better than in previous post-hoc studies that had reported a diagnostic accuracy of 90-92\% [15, 16], increasing to $93 \%$ in the current study. This observation can be likely explained by a more careful and dedicated OCT acquisition, specifically aiming to optimise the quality of OFR computation [20]. The consistent accuracy of OFR used on a prospective fashion is of paramount importance to outline the clinical usefulness and the limitations of this novel technology for routine use in the cathlab. As expected, morphofunctional methods outweighed the diagnostic performance of purely morphological methods, like MLA. Measuring MLA to assess 
the severity of coronary stenosis was indeed common practice in the past, but this approach is currently discouraged [13]. Relying on the area in just a cross-section is probably too simplistic and disregards many other parameters (lesion length, reference vessel areas, flow, microvascular resistance, amount of subtended myocardium, excentricity of the lesion, etc.) that play a crucial role in determining the functional relevance of a stenosis and can be however integrated in both virtual and wirebased physiology [26]. Remarkably, OFR showed outstanding accuracy in challenging scenarios like bifurcations or sequential lesions, confirming the validity of the computational method in these specific settings $[12,27]$.

Among morphofunctional methods, OFR tended to show better diagnostic performance than QFR (AUC 0.95 vs. 0.91, p = 0.115), in line with the retrospective studies [16]. The lack of statistical significance can be explained by the difference in sample size between both studies, as AUC values are consistently similar. This prospective study was powered to estimate the AUC in OFR rather than for a comparison vs. QFR, that was only an exploratory secondary objective. Both morphofunctional methods rely on an accurate reconstruction of the coronary anatomy to enable a precise computation of physiology. Nonetheless, angiography-based methods like QFR share all the intrinsic limitations of angiography itself, namely foreshortening, vessel overlap or calcium interfering with adequate edge detection. Conversely, OFR can overcome all these limitations and render high-resolution luminograms, thus offering a clear advantage for an optimal computation of physiology that can be instrumental in some particular cases.

Pullback speed during the OCT acquisition had negligible effect on computation of OFR. The diagnostic accuracy remained excellent irrespective of the pullback speed, provided the same coronary segment was imaged. High pullback speed $(36 \mathrm{~mm} / \mathrm{s})$ is however preferred because it allows longer pullbacks and less contrast, so the computation takes into account the true luminogram in a longer coronary segment that is otherwise assumed as physiologically inert in the lower speed (shorter pullback).

The possibility to integrate physiology within an ordinary OCT catheter opens new perspectives in interventional cardiology. It will be reassuring for QFR in case of challenging borderline lesions. Moreover, OFR permits the operator to conform to the highest standards currently recommended by guidelines, while complying with most of reim- bursement policies in developed countries, where a single adjuvant tool for PCI can be invoiced. A single catheter can be used to assess the functional severity of all pertinent lesions, other morphological features (ruptured plaque, dissection, etc.) and finally to guide and optimise PCI. This is particularly interesting for the assessment of lesions with a high priori likelihood of PCI. Finally, OFR does not require hyperemia, like other computational methods [18], sparing the patient discomfort and circumventing the contraindications of vasodilator drugs.

\section{Limitations of the study}

This is a pivotal general study of agreement performed on an unselected real-world population of patients with an indication for FFR. The impact of microvascular resistance, plaque composition, collaterals and other relevant variables on the accuracy of OFR needs to be understood in future dedicated studies. The feasibility reported for OFR refers to patients with a coronary anatomy considered a priori suitable for OCT imaging by the operator.

All the OFR computations in this study were performed offline in a central corelab. This approach optimises the performance of the new method and guarantees methodological homogeneity. The diagnostic performance of OFR calculated on-site by trained local staff of the cathlab is hitherto unknown and will require specific evaluation in further dedicated studies.

\section{Conclusions}

The prospective computation of OFR is feasible and reproducible in a real-world series, resulting in excellent agreement with FFR and better diagnostic performance than angiography-based morphofunctional methods or than a MLA cut-off, irrespective of the pullback speed used in the OCT acquisition.

\section{Impact on daily practice}

Optical flow ratio appears as the most accurate morphofunctional method hitherto to estimate FFR. This enables the integration of accurate physiology and high-resolution imaging within a single catheter. This information is instrumental for modern PCI guidance, in order to comply with current evidence-supported and guidelines-recommended standards, as with the principles of personalised medicine. Morphofunctional methods allow the reconcilement between precision PCI and the reimbursement policy of many countries, where a single adjuvant tool for PCI can be invoiced. 


\section{Acknowledgements}

Shengxian $\mathrm{Tu}$ would like to acknowledge the support by the National Key Research and Development Program of China (Grant No. 2016YFC0100500), the Natural Science Foundation of China (Grant No. 81871460), and by the Program of Shanghai Technology Research Leader.

Conflict of interest: The authors have declared no conflict of interest in relation to this study, with the exception that Shengxian Tu received research support from Pulse medical imaging technology. Other authors report no conflicts of interest regarding this manuscript.

\section{References}

1. De Bruyne B, Pijls NHJ, Kalesan B, et al. Fractional flow reserve-guided PCI versus medical therapy in stable coronary disease. N Engl J Med. 2012; 367(11): 991-1001, doi: 10.1056/ NEJMoa1205361, indexed in Pubmed: 22924638.

2. Tonino PAL, De Bruyne B, Pijls NHJ, et al. FAME Study Investigators. Fractional flow reserve versus angiography for guiding percutaneous coronary intervention. N Engl J Med. 2009; 360(3): 213-224, doi: 10.1056/NEJMoa0807611, indexed in Pubmed: 19144937.

3. Bech GJ, De Bruyne B, Pijls NH, et al. Fractional flow reserve to determine the appropriateness of angioplasty in moderate coronary stenosis: a randomized trial. Circulation. 2001; 103(24): 2928-2934, doi: 10.1161/01.cir.103.24.2928, indexed in Pubmed: 11413082 .

4. Toth GG, Toth B, Johnson NP, et al. Revascularization decisions in patients with stable angina and intermediate lesions: results of the international survey on interventional strategy. Circ Cardiovasc Interv. 2014; 7(6): 751-759, doi: 10.1161/CIRCINTERVENTIONS.114.001608, indexed in Pubmed: 25336468.

5. Härle T, Zeymer U, Hochadel M, et al. Real-world use of fractional flow reserve in Germany: results of the prospective ALKK coronary angiography and PCI registry. Clin Res Cardiol. 2017; 106(2): 140-150, doi: 10.1007/s00392-016-1034-5, indexed in Pubmed: 27599974.

6. Lee HS, Lee JM, Nam CW, et al. Consensus document for invasive coronary physiologic assessment in Asia-Pacific countries. Cardiol J. 2019; 26(3): 215-225, doi: 10.5603/CJ.a2019.0054, indexed in Pubmed: 31225632.

7. Gutiérrez-Chico JL, Regar E, Nüesch E, et al. Delayed coverage in malapposed and side-branch struts with respect to well-apposed struts in drug-eluting stents: in vivo assessment with optical coherence tomography. Circulation. 2011; 124(5): 612-623, doi: 10.1161/CIRCULATIONAHA.110.014514, indexed in Pubmed: 21768536.

8. Gutiérrez-Chico JL, Wykrzykowska J, Nüesch E, et al. Vascular tissue reaction to acute malapposition in human coronary arteries: sequential assessment with optical coherence tomography. Circ Cardiovasc Interv. 2012; 5(1): 20-29, S1, doi: 10.1161/CIRCINTERVENTIONS.111.965301, indexed in Pubmed: 22319063.

9. Gutiérrez-Chico JL, Alegría-Barrero E, Teijeiro-Mestre R, et al. Optical coherence tomography: from research to practice. Eur
Heart J Cardiovasc Imaging. 2012; 13(5): 370-384, doi: 10.1093/ ehjci/jes025, indexed in Pubmed: 22330231.

10. Ali ZA, Maehara A, Généreux P, et al. ILUMIEN III: OPTIMIZE PCI Investigators. Optical coherence tomography compared with intravascular ultrasound and with angiography to guide coronary stent implantation (ILUMIEN III: OPTIMIZE PCI): a randomised controlled trial. Lancet. 2016; 388(10060): 2618-2628, doi: 10.1016/S0140-6736(16)31922-5, indexed in Pubmed: 27806900.

11. Gonzalo N, Escaned J, Alfonso F, et al. Morphometric assessment of coronary stenosis relevance with optical coherence tomography: a comparison with fractional flow reserve and intravascular ultrasound. J Am Coll Cardiol. 2012; 59(12): 1080-1089, doi: 10.1016/j.jacc.2011.09.078, indexed in Pubmed: 22421301.

12. Li Y, Gutiérrez-Chico JL, Holm NR, et al. Impact of side branch modeling on computation of endothelial shear stress in coronary artery disease: coronary tree reconstruction by fusion of 3D angiography and OCT. J Am Coll Cardiol. 2015; 66(2): 125-135, doi: 10.1016/j.jacc.2015.05.008, indexed in Pubmed: 26160628.

13. Neumann FJ, Sousa-Uva M, Ahlsson A, et al. 2018 ESC/EACTS Guidelines on myocardial revascularization. Eur Heart J. 2018; 40(2): 87-165, doi: 10.1093/eurheartj/ehy394.

14. Tian F, Yu W, Huang J, et al. First presentation of integration of intravascular optical coherence tomography and computational fractional flow reserve. Int J Cardiovasc Imaging. 2019; 35(4): 601-602, doi: 10.1007/s10554-018-1491-1, indexed in Pubmed: 30390169.

15. Yu W, Huang J, Jia D, et al. Diagnostic accuracy of intracoronary optical coherence tomography-derived fractional flow reserve for assessment of coronary stenosis severity. EuroIntervention. 2019; 15(2): 189-197, doi: 10.4244/EIJ-D-19-00182, indexed in Pubmed: 31147309.

16. Huang J, Emori H, Ding D, et al. Comparison of diagnostic performance of intracoronary optical coherence tomography-based and angiography-based fractional flow reserve for evaluation of coronary stenosis. EuroIntervention. 2020 [Epub ahead of print], doi: 10.4244/EIJ-D-19-01034, indexed in Pubmed: 31951207.

17. Tu S, Barbato E, Köszegi $Z$, et al. Fractional flow reserve calculation from 3-dimensional quantitative coronary angiography and TIMI frame count: a fast computer model to quantify the functional significance of moderately obstructed coronary arteries. JACC Cardiovasc Interv. 2014; 7(7): 768-777, doi: 10.1016/j. jcin.2014.03.004, indexed in Pubmed: 25060020.

18. Tu S, Westra J, Yang J, et al. Diagnostic accuracy of fast computational approaches to derive fractional flow reserve from diagnostic coronary angiography: the international multicenter FAVOR pilot study. JACC Cardiovasc Interv. 2016; 9(19): 2024-2035, doi: 10.1016/j.jcin.2016.07.013, indexed in Pubmed: 27712739.

19. Prati F, Cera M, Ramazzotti V, et al. Safety and feasibility of a new non-occlusive technique for facilitated intracoronary optical coherence tomography (OCT) acquisition in various clinical and anatomical scenarios. EuroIntervention. 2007; 3(3): 365-370, doi: 10.4244/eijv3i3a66, indexed in Pubmed: 19737719.

20. Gutiérrez-Chico JL, Cortés C, Schincariol M, et al. A formula to calculate the contrast volume required for optimal imaging quality in optical coherence tomography with non-occlusive technique. Cardiol J. 2018; 25(5): 574-581, doi: 10.5603/ CJ.a2018.0112, indexed in Pubmed: 30246237.

21. Xu B, Tu S, Qiao S, et al. Diagnostic accuracy of angiography-based quantitative flow ratio measurements for online assessment of 
coronary stenosis. J Am Coll Cardiol. 2017; 70(25): 3077-3087, doi: 10.1016/j.jacc.2017.10.035, indexed in Pubmed: 29101020.

22. Westra J, Andersen BK, Campo G, et al. Diagnostic performance of in-procedure angiography-derived quantitative flow reserve compared to pressure-derived fractional flow reserve: the FAVOR II Europe-Japan study. J Am Heart Assoc. 2018; 7(14), doi: 10.1161/JAHA.118.009603, indexed in Pubmed: 29980523.

23. Karanasos A, Tu S, van Ditzhuijzen NS, et al. A novel method to assess coronary artery bifurcations by OCT: cut-plane analysis for side-branch ostial assessment from a main-vessel pullback. Eur Heart J Cardiovasc Imaging. 2015; 16(2): 177-189, doi: 10.1093/ehjci/jeu176, indexed in Pubmed: 25227268.

24. Finet G, Gilard M, Perrenot B, et al. Fractal geometry of arterial coronary bifurcations: a quantitative coronary angiography and intravascular ultrasound analysis. EuroIntervention. 2008; 3(4): 490-498, doi: 10.4244/eijv3i4a87, indexed in Pubmed: 19736093.

25. Liao JJZ. Sample size calculation for an agreement study. Pharm Stat. 2010; 9(2): 125-132, doi: 10.1002/pst.382, indexed in Pubmed: 19507134

26. Gutiérrez-Chico JL, Zhao S, Chatzizisis YS. Vorticity: At the crossroads of coronary biomechanics and physiology. Atherosclerosis. 2018; 273: 115-116, doi: 10.1016/j.atherosclerosis.2018.04.001, indexed in Pubmed: 29665968.

27. Gutierrez-Chico JL, Cortes C, Jaguszewski M, et al. A simplified formula to calculate fractional flow reserve in sequential lesions circumventing the measurement of coronary wedge pressure: The APIS-S pilot study. Cardiol J. 2019; 26(4): 310-321, doi: 10.5603/CJ.a2019.0067, indexed in Pubmed: 31257567. 